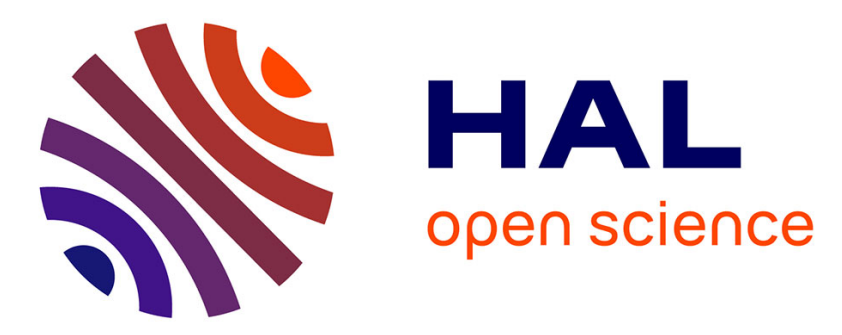

\title{
Robust global synchronization of Brockett oscillators
}

\author{
Hafiz Ahmed, Rosane Ushirobira, Denis Efimov
}

\section{To cite this version:}

Hafiz Ahmed, Rosane Ushirobira, Denis Efimov. Robust global synchronization of Brockett oscillators. IEEE Transactions on Control of Network Systems, 2018, pp.1-10. 10.1109/tcns.2018.2813927 . hal01965539

\section{HAL Id: hal-01965539 \\ https://hal.inria.fr/hal-01965539}

Submitted on 26 Dec 2018

HAL is a multi-disciplinary open access archive for the deposit and dissemination of scientific research documents, whether they are published or not. The documents may come from teaching and research institutions in France or abroad, or from public or private research centers.
L'archive ouverte pluridisciplinaire HAL, est destinée au dépôt et à la diffusion de documents scientifiques de niveau recherche, publiés ou non, émanant des établissements d'enseignement et de recherche français ou étrangers, des laboratoires publics ou privés. 


\title{
Robust global synchronization of Brockett oscillators
}

\author{
Hafiz Ahmed, Member, IEEE, Rosane Ushirobira, Denis Efimov, Senior Member, IEEE
}

\begin{abstract}
In this article, motivated by a recent work of R. Brockett [1], a robust global synchronization problem of multistable Brockett oscillators has been studied within an Input-to-State Stability (ISS) framework. Two synchronization protocols are designed with respect to compact invariant sets of the unperturbed Brockett oscillator. The conditions obtained in our work are global and applicable to families of non-identical oscillators in contrast to the local analysis of [1]. Numerical simulation examples illustrate our theoretical results.
\end{abstract}

Index Terms-Input-to-State Stability, synchronization, multistability, Brockett oscillator.

\section{INTRODUCTION}

Over the past decade, considerable attention has been devoted to the problem of coordinated motion of multiple autonomous agents due to its broad applications in various areas. One critical issue related with multi-agent systems is to develop distributed control policies based on local information that enables all agents to reach an agreement on certain quantities of interest, which is known as the consensus problem [2]-[4] (e.g. formation control [5], [6], attitude synchronization [7]). A classic example of distributed coordination/consensus in physics, engineering and biology is the synchronization of arrays of coupled nonlinear oscillators [8]-[16]. Oscillators synchronization has several potential application domains, for instance in power networks [17]-[22], smooth operations of micro-grids, real-time distributed control in networked systems [23] and so on.

The problem of synchronization has been addressed by researchers from various technical fields like physics, biology, neuroscience, automatic control, etc. To have a better insight on the contribution of automatic control community in this area, interested readers may consult [24]-[30]. In the context of the synchronization of oscillators, R. Brockett has recently introduced the following model [1]:

$$
\ddot{x}+\varepsilon \dot{x}\left(\dot{x}^{2}+x^{2}-1\right)+x=\varepsilon^{2} u,
$$

where $x \in \mathbb{R}^{n}$ is the state, $u \in \mathbb{R}^{n}$ is the control and $\varepsilon>0$ is a parameter. In [1], a centralized synchronization

H. Ahmed is with the School of Mechanical, Aerospace and Automotive Engineering, Coventry University, The Futures Institute, Coventry CV1 2TL, United Kingdom (e-mail: ac7126@ coventry.ac.uk).

R. Ushirobira and D. Efimov are with Inria, Non-A team, 40 avenue Halley, 59650 Villeneuve d'Ascq, France. D. Efimov is also with CRIStAL CNRS UMR 9189, Ecole Centrale de Lille, Avenue Paul Langevin, 59651 Villeneuve d'Ascq, France. E-mail: \{rosane.ushirobira, denis.efimov@inria.fr\}.

The project is partly supported by ANR project WaQMoS (ANR 15 CE 04 0002). H. Ahmed was partly supported by the regional council of Nord-Pas de Calais, France during the period of this work.

The work has been performed while H. Ahmed was part of the NON-A team. protocol has been proposed for the model (1), such that the conventional averaging theory does not predict the existence of a periodic (almost periodic) solution for small $\varepsilon$. However, a qualitative synchronization together with a small amplitude irregular motion can be observed through numerical studies. Following [1], for $\varepsilon$ sufficiently small, but non-zero, let us introduce the set

$$
\begin{aligned}
S_{\varepsilon}= & \left\{(x, \dot{x}) \in \mathbb{R}^{2}: \dot{x}^{2}+x^{2}-1\right. \\
& \left.+2 \varepsilon^{2} x \dot{x} \operatorname{sign}\left(\dot{x}^{2}+x^{2}-1\right)=\varepsilon\right\},
\end{aligned}
$$

which contains two smooth closed contours: $\Gamma_{\varepsilon}^{+}$lies outside the unit circle in the $(x, \dot{x})$-space and $\Gamma_{\varepsilon}^{-}$lies inside the unit circle. Both curves approach the unit circle as $\varepsilon$ goes to zero. Then the main result of [1] is given below.

Theorem 1. Let $\Gamma_{\varepsilon}^{ \pm}$be as before. Then there exist $\varepsilon_{0}>0$ such that for all $0<\varepsilon<\varepsilon_{0}$, the solutions of (1) beginning in the annulus bounded by $\Gamma_{\varepsilon}^{+}$and $\Gamma_{\varepsilon}^{-}$remain in this annulus for all time, provided that $|u| \leq \sqrt{x^{2}+\dot{x}^{2}}$.

Theorem 1 provides a local synchronization result which depends on a small parameter $\varepsilon \neq 0$. Moreover, the result is applicable to the synchronization of identical oscillators only.

The goal of this work is to extend the result of [1] and to develop a protocol of global synchronization in the network of (1), for the case of identical and non-identical models of the agents ${ }^{1}$. It is assumed that the oscillators are connected through a $N$-cycle graph $^{2}$ [32]. The proposed solution is based on the framework of input-to-state stability (ISS) for multistable systems [33].

The ISS property provides a natural framework of stability analysis with respect to input perturbations (see [34] and references therein). The classical definition allows the stability properties with respect to arbitrary compact invariant sets (and not simply equilibria) to be formulated and characterized. Nevertheless, the implicit requirement is that these sets should be simultaneously Lyapunov stable and globally attractive, which makes the basic theory not applicable for a global analysis of many dynamical behaviors of interest having multistability [35]-[37], periodic oscillations [38], just to name a few, and only a local analysis remains possible [39]. Some attempts were made to overcome such limitations by introducing the notions of almost global stability [40] and almost input-tostate stability [41], etc.

Recently, the authors in [33] have found that a natural way of developing ISS theory for systems with multiple invariant

\footnotetext{
${ }^{1}$ Part of the results has been presented in [31].

${ }^{2}$ A cycle graph $C_{N}$ is a graph on $N$ nodes containing a single cycle through all nodes, or in other words, $N$ number of vertices connected in a closed chain.
} 
sets consists in relaxing the Lyapunov stability requirement [42] (rather than the global nature of the attractivity property). Using this relatively mild condition, the ISS theory has been generalized in [33], as well as the related literature on time-invariant autonomous dynamical systems on compact spaces [43] for multistable systems. Multistability accounts for the possible coexistence of various oscillatory regimes or equilibria in the state space of the system for the same set of parameters. Any system that exhibits multistability is called a multistable system. Frequently, for a given set of initial conditions and inputs it is very difficult to predict the asymptotic regime that a multistable system will attain asymptotically [44]. Following the results of [33], the authors in [45] have provided conditions for the robust synchronization of multistable systems in the presence of external inputs. Readers can consult [46] for an overview of recent developments in the ISS framework, dealing in particular with the extension of the classical concept to systems with multiple invariant sets and possibly evolving on Riemannian manifolds.

Main contribution: The results presented in [45] can be applied to provide sufficient conditions for the existence of robust synchronization for identical/non-identical Brockett oscillators in the presence of external inputs under some mild assumptions. In [31], a global synchronization protocol has been proposed for the case $N=2$ avoiding additional hypothesis. In this work, this result is extended to the general case $N>2$ and to this end another synchronization control is proposed, which is not based on the theory of [45] and a special Lyapunov function is designed characterizing synchronization conditions for a family of non-identical Brockett oscillators. In opposite to the local results of [1], the conditions obtained in this work are global. The obtained synchronized system may demonstrate phase or anti-phase synchronization phenomena depending on parameters of the oscillators. The results obtained in this work are based on the assumption that the oscillators are connected through $N$-cycle graph. However, other network topologies may also be considered.

The rest of this paper is organized as follows. Some preliminaries about the robust synchronization of multistable systems can be found in Section II. Details about Brockett oscillators (such as the proof that they possess ISS property) and the synchronization of a family of oscillators (the main results) can be found in sections III and IV, respectively. In Section $\mathrm{V}$, a numerical simulation example is given to illustrate these results. Concluding remarks in Section VI close this article.

\section{PREliminaries: Robust SYNCHRONIZATION OF MULTISTABLE SYSTEMS}

Let us consider the following family of nonlinear systems:

$$
\dot{x}_{i}(t)=f_{i}\left(x_{i}(t), u_{i}(t), d_{i}(t)\right), i=\overline{1, N}, N>1,
$$

where the state $x_{i}(t) \in M_{i} \quad\left(M_{i}\right.$ is $n_{i}$-dimensional $\mathcal{C}^{2}$ connected and orientable Riemannian manifold without a boundary), the control $u_{i}(t) \in \mathbb{R}^{m_{i}}$ and the external disturbance $d_{i}(t) \in \mathbb{R}^{p_{i}}\left(u_{i}(\cdot)\right.$ and $d_{i}(\cdot)$ are locally essentially bounded and measurable signals) for $t \geq 0$; and the map $f_{i}: M_{i} \times \mathbb{R}^{m_{i}} \times \mathbb{R}^{p_{i}} \rightarrow T_{x_{i}} M_{i}$ is $\mathcal{C}^{1}, f_{i}(0,0,0)=0$. Denote the common state vector of (2) as $x=\left[x_{1}^{T}, \ldots, x_{N}^{T}\right]^{T} \in M=$ $\prod_{i=1}^{N} M_{i}$, so $M$ is the corresponding Riemannian manifold of dimension $n=\sum_{i=1}^{N} n_{i}$ where the family (2) evolves and $d=\left[d_{1}^{T}, \ldots, d_{N}^{T}\right]^{T} \in \mathbb{R}^{p}$ with $p=\sum_{i=1}^{N} p_{i}$ is the exogenous input. In this section, we recall the result on robust synchronization of multistable systems obtained in [45].

Assumption 1. For all $i=\overline{1, N}$, each system in (2) has a compact invariant set $\mathcal{W}_{i}$ containing all $\alpha-$ and $\omega$-limit sets of $\dot{x}_{i}(t)=f_{i}\left(x_{i}(t), 0,0\right), \mathcal{W}_{i}$ is decomposable in the sense of Definition 3 given in [31], and the system is ISS with respect to the set $\mathcal{W}_{i}$ and the inputs $u_{i}$ and $d_{i}$.

Details about ISS w.r.t. decomposable invariant sets can be found in [33]. Assumption 1 implies that family (2) is composed of robustly stable nonlinear systems.

Let a $\mathcal{C}^{1}$ function $\psi(x): M \rightarrow \mathbb{R}^{q}, \psi(0)=0$ be a synchronization measure for (2). We say that the family (2) is synchronized (or reached the consensus) if $\psi(x(t)) \equiv 0$ for all $t \geq 0$ on the solutions of the network under properly designed control actions

$$
u_{i}(t)=\varphi_{i}(\psi(x(t)))
$$

$\left(\varphi_{i}: \mathbb{R}^{q} \rightarrow \mathbb{R}^{m_{i}}\right.$ is a $\mathcal{C}^{1}$ function, $\left.\varphi_{i}(0)=0\right)$ for $d(t) \equiv 0$, $t \geq 0$. In this case, the set $\mathcal{A}=\{x \in \mathcal{W} \mid \psi(x)=0\}$ contains the synchronous solutions of the unperturbed family in (2) and the problem of synchronization of "natural" trajectories is considered since $\mathcal{A} \subset \mathcal{W}$ (due to $\varphi_{i}(0)=0$ in (3), the convergence of $\psi$ (synchronization/consensus) implies that the solutions belong to $\mathcal{W}$ ).

The main result of [45] states that by selecting the shapes of $\varphi_{i}$, it is possible to guarantee robust synchronization of (2) for any measurable and essentially bounded input $d$.

Proposition 2. Let Assumption 1 be satisfied for (2). Then there exist $\varphi_{i}, i=\overline{1, N}$ in (3) such that the interconnection (2), (3) has practical global stability ( $p G S$ ) property (see the formal definition in [45]) with respect to the set $\mathcal{W}$.

For example, the result of Proposition 2 is valid for any bounded functions $\varphi_{i}, i=\overline{1, N}$ in (3).

Assumption 2. The set $\mathcal{A}$ is compact, it contains all $\alpha$ - and $\omega$-limit sets of (2), (3) for $d=0$, and it is decomposable.

Here additionally, it is assumed that the controls $\varphi_{i}(\psi)$ ensure the network global synchronization, while the decomposability in general follows from Assumption 1.

Theorem 3. Let conditions of Proposition 2 be satisfied together with Assumption 2, then the interconnection (2), (3) is ISS with respect to $\mathcal{A}$.

\section{PROPERTIES OF THE BROCKETT OSCILLATOR}

Let us consider the Brockett oscillator [1]:

$$
\ddot{\xi}+b \dot{\xi}\left(\dot{\xi}^{2}+\xi^{2}-1\right)+\xi=a u,
$$

where $\xi \in \mathbb{R}, \dot{\xi} \in \mathbb{R}$ are the states variables, $a, b>0$ are parameters and $u$ is the control input. By considering $x_{1}=\xi$, 
$\dot{x}_{1}=x_{2}=\dot{\xi}, x=\left[x_{1}, x_{2}\right]^{T}$ and $|x|=\sqrt{x_{1}^{2}+x_{2}^{2}}$ equation (4) can be written in the state-space form as:

$$
\begin{aligned}
& \dot{x}_{1}=x_{2} \\
& \dot{x}_{2}=-x_{1}+a u-b x_{2}\left(|x|^{2}-1\right),
\end{aligned}
$$

where the state of the system (5), i.e. $x$, evolves in $M=\mathbb{R}^{2}$. By analyzing equation (5) it can be seen that the unperturbed system admits two invariant sets: namely, the origin $\mathcal{W}_{1}=\{0\}$ and the limit cycle $\mathcal{W}_{2}=\Gamma=\left\{x \in M:|x|^{2}=1\right\}$. So, the invariant set for the trajectories of (5) can be defined as:

$$
\mathcal{W}:=\mathcal{W}_{1} \cup \mathcal{W}_{2}=\{0\} \cup \Gamma \text {. }
$$

In order to verify the decomposability of the invariant set $\mathcal{W}$, we need to know the nature of the equilibrium $\mathcal{W}_{1}$ and the limit cycle $\mathcal{W}_{2}=\Gamma$. This information can be obtained by analyzing the Lyapunov stability of the unperturbed system (5).

\section{A. Stability of the autonomous Brockett oscillator}

Since $\mathcal{W}$ is invariant for the trajectories of (6), then the following proposition provides the stability of the unforced Brockett oscillator ((5) with $u=0)$ with respect to $\mathcal{W}$.

Proposition 4. For (5) with $u=0$, the limit cycle $\Gamma$ is almost globally asymptotically stable and the origin is unstable.

Proof: The instability of the origin of the unperturbed system (5) can be verified for a linearized version of the system. The eigenvalues of the linearized system $\lambda_{1,2}=$ $\frac{1}{2}\left(b \pm \sqrt{b^{2}-4}\right)$ have always positive real parts for any $b>0$. Alternatively, this fact can also be checked through an LMI formulation which is given in Remark 5.

To analyze the stability of the limit cycle $\mathcal{W}_{2}$, let us consider the following Lyapunov function:

$$
U(x)=\frac{1}{2}\left(|x|^{2}-1\right)^{2}
$$

which is zero on the set $\mathcal{W}_{2}$ and positive otherwise. Evaluating the total derivative of $U$ along the solutions of (5), we obtain:

$$
\begin{aligned}
\dot{U} & =\left(|x|^{2}-1\right)\left\{2 a u x_{2}-2 b x_{2}^{2}\left(|x|^{2}-1\right)\right\} \\
& =-2 b x_{2}^{2}\left(|x|^{2}-1\right)^{2}+2 a u x_{2}\left(|x|^{2}-1\right) \\
& \leq-2 b x_{2}^{2}\left(|x|^{2}-1\right)^{2}+b x_{2}^{2}\left(|x|^{2}-1\right)^{2}+\frac{a^{2}}{b} u^{2} \\
& =-b x_{2}^{2}\left(|x|^{2}-1\right)^{2}+\frac{a^{2}}{b} u^{2} .
\end{aligned}
$$

Then for $u=0$ we have $\dot{U} \leq 0$ and all trajectories are globally bounded. By LaSalle's invariance principle [47], all trajectories of the system converge to the set where $\dot{U}=0$. Note that $\{x \in M: \dot{U}=0\}=\mathcal{W}_{2} \cup\left\{x \in M: x_{2}=0\right\}$ and on the line $x_{2}$ there is the only invariant solution at the origin (in $\mathcal{W}_{1}$ ), therefore $\dot{U}=0$ for all $x \in \mathcal{W}$, which contains all invariant solutions of the system. Since the origin is unstable, it can be concluded that the limit cycle $\mathcal{W}_{2}$ is almost globally asymptotically stable.

Remark 5. To check the instability of the origin in an alternative way, let us consider a small closed ball with the radius $\rho>0$ around the origin $B(\rho)=\left\{x \in \mathbb{R}^{2}:|x|^{2} \leq \rho\right\}$. Inside this ball, by imposing the parameter $b=1$ without loosing generality, the unperturbed system of (5) can be written as the following uncertain linear system:

$$
\dot{x}=A x, A=\left[\begin{array}{cc}
0 & 1 \\
-1 & -(\tilde{\rho}-1)
\end{array}\right], \tilde{\rho} \in[0, \rho],
$$

where the matrix $A \in \mathbb{R}^{2 \times 2}$ belongs to the domain $\mathcal{D}_{A}$ defined as:

$$
\mathcal{D}_{A} \triangleq\left\{A: A=\beta_{1} A_{1}+\beta_{2} A_{2}, \beta_{1}, \beta_{2}>0, \sum_{i=1}^{2} \beta_{i}=1\right\}
$$

with $A_{1}=\left[\begin{array}{cc}0 & 1 \\ -1 & 1\end{array}\right]$ and $A_{2}=\left[\begin{array}{cc}0 & 1 \\ -1 & -(\rho-1)\end{array}\right]$. Then, by applying Chetaev instability theorem [48], it can be concluded that the origin is unstable if there exist $P>0, Q>0$ such that for $i=1,2$

$$
A_{i}^{T} P+P A_{i} \succeq Q .
$$

The LMI (9) can be easily verified by using any standard solvers like Yalmip [49]. For example, let us select $\rho=0.2$, then the following values are obtained satisfying LMI (9):

$$
P=\left[\begin{array}{cc}
21.4643 & -6.8278 \\
\star & 17.8390
\end{array}\right], Q=\left[\begin{array}{cc}
6.1040 & -1.3080 \\
\star & 7.8838
\end{array}\right]
$$

As a result, it can be concluded that the origin is unstable.

\section{B. Stability of the non-autonomous Brockett oscillator}

In the previous section, we have proved the stability of the unperturbed system with $u=0$. In this section, we will analyze the stability of the Brockett oscillator in the presence of input $u$. As it was shown in the previous section, $\mathcal{W}$ contains all $\alpha-$ and $\omega$-limit sets of the unperturbed system in (5), and it admits a decomposition without cycles. Consequently the result of [33] can be applied to show the robust stability of the Brockett oscillator in (5) with respect to $\mathcal{W}$ :

Proposition 6. The system (5) is ISS with respect to the set W.

Proof: To prove the ISS property, let us introduce two new variables $y$ and $h$ as,

$$
\begin{aligned}
y(x) & =|x|^{2}-1, \dot{y}=-2 b x_{2}^{2} y+2 a x_{2} u \\
h(x) & =\left(x_{1}+x_{2}\right) y, \dot{h}=a\left[y+2 x_{2}\left(x_{1}+x_{2}\right)\right] u \\
& -\left(h-2 x_{2} y+b x_{2} y^{2}+2 b x_{2}^{2} h\right) .
\end{aligned}
$$

Next, let us consider the following Lyapunov function for (5) with some $c, d>0$ :

$$
W(x)=\frac{1}{2}\left(h^{2}(x)+c y^{2}(x)+\frac{1}{2} d y^{4}(x)\right) .
$$

Notice that $W(x)=0$ for all $x \in \mathcal{W}_{2}$ and positive otherwise. Therefore, there exist $\alpha_{1}, \alpha_{2} \in \mathcal{K}_{\infty}$ such that the condition $\alpha_{1}\left(|x|_{\Gamma}\right) \leq W(x) \leq \alpha_{2}\left(|x|_{\Gamma}+c\right)$ is satisfied for all $x \in M$ for the above function $W(x)$ for some $c \geq 0$, and where $|x|_{\Gamma}=\inf _{y \in \Gamma}|x-y|$ is the distance to the set $\Gamma$ from a 
point $x \in M$. Evaluating the total derivative of $W$, along the solutions of (5), we obtain

$$
\begin{aligned}
\dot{W}= & c y \dot{y}+h \dot{h}+d y^{3} \dot{y} \\
= & 2 a u\left[h\left(x_{2}^{2}+x_{1} x_{2}+0.5 y\right)+x_{2} y\left(c+d y^{2}\right)\right] \\
& -h^{2}-2 b x_{2}^{2}\left[h^{2}+y^{2}\left(c+d y^{2}\right)\right]+h x_{2} y(2-b y) .
\end{aligned}
$$

Next by applying Young's inequality, we can derive the series of relations:

$$
\begin{gathered}
2 h x_{2} y \leq \frac{1}{2} h^{2}+2 x_{2}^{2} y^{2}, h x_{2} y^{2} \leq \frac{h^{2}}{4 b}+b x_{2}^{2} y^{4}, \\
x_{2}^{2} h u \leq x_{2}^{2}\left(\frac{b}{4 a} h^{2}+\frac{a}{b} u^{2}\right), \\
h u x_{1} x_{2} \leq \frac{b}{4 a} h^{2} x_{2}^{2}+\frac{a}{b} x_{1}^{2} u^{2}, h u y \leq \frac{h^{2}}{16 a}+4 a y^{2} u^{2}, \\
\left(c+d y^{2}\right) u x_{2} y \leq\left(c+d y^{2}\right)\left(\frac{b}{2 a} x_{2}^{2} y^{2}+\frac{a}{2 b} u^{2}\right) .
\end{gathered}
$$

By substituting these inequalities for $c=\frac{3}{b}, d=2 b$ and after simplification, we obtain

$$
\begin{aligned}
\dot{W} \leq & -h^{2}\left(\frac{3}{16}+b x_{2}^{2}\right)-x_{2}^{2} y^{2}\left(1+b^{2} y^{2}\right) \\
& +\frac{a^{2}}{b^{2}}\left[2 b|x|^{2}+6 b^{2} y^{2}+3\right] u^{2} .
\end{aligned}
$$

From the properties of the functions $h$ and $y$ we can substantiate that

$$
\frac{3}{16} h^{2}+x_{2}^{2} y^{2} \geq \frac{5-\sqrt{17}}{8}|x|^{2} y^{2},
$$

and $W$ is a practical ISS Lyapunov function for (5) since

$$
\begin{gathered}
|x|^{2} \geq \max \left\{q, 16 \frac{2 b q+6 b^{2}(q-1)^{2}+3}{(5-\sqrt{17})(q-1)^{2}} \frac{a^{2}}{b^{2}} u^{2}\right\} \\
\Rightarrow \dot{W} \leq-\frac{5-\sqrt{17}}{16}|x|^{2} y^{2}
\end{gathered}
$$

for any $q>1$. Consequently, using Theorem 1 (given in [33]) it can be concluded that the system (5) is ISS with respect to the set $\mathcal{W}$ from the input $u$.

Remark 7. It is straightforward to check that there exists a function $\alpha \in \mathcal{K}_{\infty}$ such that for all $x \in M$ and $u=0$ we have $\dot{W} \leq-\alpha\left(|x|_{\mathcal{W}}\right)$. Thus $W$ is a global Lyapunov function establishing multistability of (5) with respect to $\mathcal{W}$ for $u=0$.

\section{SYNCHRONIZATION OF BROCKETT OSCILlATORS}

The following family of Brockett oscillators is considered in this section for some $N>1$ :

$$
\begin{aligned}
& \dot{x}_{1 i}=x_{2 i}, \\
& \dot{x}_{2 i}=a_{i} u_{i}-x_{1 i}-b_{i} x_{2 i}\left(\left|x_{i}\right|^{2}-1\right), i=\overline{1, N},
\end{aligned}
$$

where $a_{i}, b_{i}>0$ are the parameters of an individual oscillator, the state $x_{i}=\left[\begin{array}{ll}x_{1 i} & x_{2 i}\end{array}\right]^{T} \in M_{i}=\mathbb{R}^{2}$, the control $u_{i} \in \mathbb{R}\left(u_{i}: \mathbb{R}_{+} \rightarrow \mathbb{R}\right.$ is locally essentially bounded and measurable signal). Denote the common state vector of (2) as $x=\left[x_{1}^{T}, \ldots, x_{N}^{T}\right]^{T} \in M=\prod_{i=1}^{N} M_{i}$, so $M$ is the corresponding Riemannian manifold of dimension $n=2 N$ where the family (2) behaves and $u=\left[u_{1}, \ldots, u_{N}\right]^{T} \in \mathbb{R}^{N}$ is the common input. Through propositions 4 and 6 , it has been shown that each member of family (11) is robustly stable with respect to the set $\mathcal{W}_{i}=\mathcal{W}_{1 i} \cup \mathcal{W}_{2 i}$, where $\mathcal{W}_{1 i}=\{0\}$ and $\mathcal{W}_{2 i}=\left\{x_{i} \in M_{i}:\left|x_{i}\right|^{2}=1\right\}$. Consequently, the family (11) is a robustly stable nonlinear system. As a result, Assumption 1 is satisfied for the case of (11).

There are several works devoted to synchronization and design of consensus protocols for such a family or oscillatory network [50]-[52].

\section{A. Problem statement}

Let a $\mathcal{C}^{1}$ function $\psi: M \rightarrow \mathbb{R}^{q}, \psi(0)=0$ be a synchronization measure for (11). We say that the family (11) is synchronized (or reached the consensus) if $\psi(x(t)) \equiv 0$ for all $t \geq 0$ on the solutions of the network under properly designed control actions

$$
u_{i}(t)=\varphi_{i}[\psi(x(t))]
$$

where $\varphi_{i}: \mathbb{R}^{q} \rightarrow \mathbb{R}$ is a $\mathcal{C}^{1}$ function, $\varphi_{i}(0)=0$. Due to the condition $\varphi_{i}(0)=0$, the convergence of $\psi$ (synchronization/consensus) implies that the solutions of the interconnection belong to $\mathcal{W}=\prod_{i=1}^{N} \mathcal{W}_{i}$. In this case the set $\mathcal{A}=\{x \in \mathcal{W} \mid \psi(x)=0\}$ contains the synchronous solutions of the family in (12) and the problem of synchronization of "natural" trajectories is considered since $\mathcal{A} \subset \mathcal{W}$.

In this work we deal with the following synchronization measure:

$$
\begin{aligned}
\psi & =\left[\psi_{1}, \ldots, \psi_{N}\right]^{T}, \\
\psi_{i} & = \begin{cases}x_{2(i+1)}-x_{2 i}, & i=\overline{1, N-1} \\
x_{21}-x_{2 N}, & i=N\end{cases}
\end{aligned}
$$

From a graph theory point of view, the oscillators are connected through a $N$-cycle graph [32] (each oscillator needs only the information of its next neighbor), i.e.

$$
\psi=S\left[\begin{array}{c}
x_{21} \\
\vdots \\
x_{2 N}
\end{array}\right], S=\left[\begin{array}{ccccc}
-1 & 1 & 0 & \cdots & 0 \\
0 & -1 & 1 & \ddots & \\
& \ddots & \ddots & \ddots & \\
& & & & 1 \\
1 & & & & -1
\end{array}\right],
$$

and any other connection type can be studied similarly. Next, let us define the synchronization error among the various states of the oscillators as follows for $i=\overline{1, N-1}$ :

$$
e_{2 i-1}=x_{1 i}-x_{1(i+1)}, \dot{e}_{2 i-1}=x_{2 i}-x_{2(i+1)}=e_{2 i}
$$

and $e_{2 N-1}=x_{1 N}-x_{11}, \dot{e}_{2 N-1}=x_{2 N}-x_{21}=e_{2 N}$. Thus,

$$
\begin{aligned}
\psi_{i} & =-e_{2 i} \quad i=\overline{1, N} \\
\psi_{N} & =-\sum_{i=1}^{N-1} e_{2 i}
\end{aligned}
$$


and the quantity $e=\left[e_{1}, e_{2}, \ldots, e_{2 N}\right]=0$ implies that $\psi=0$ (the synchronization state is reached). For $y_{i}=\left|x_{i}\right|^{2}-1$ the error dynamics can be written in the form:

$$
\begin{aligned}
\dot{e}_{2 i-1}= & e_{2 i}, i=\overline{1, N} \\
\dot{e}_{2 i}= & -e_{2 i-1}+a_{i} u_{i}-a_{i+1} u_{i+1}-b_{i} x_{2 i} y_{i} \\
& +b_{i+1} x_{2(i+1)} y_{i+1}, i=\overline{1, N-1}, \\
\dot{e}_{2 N}= & -e_{2 N-1}+a_{N} u_{N}-a_{1} u_{1}-b_{N} x_{2 N} y_{N} \\
& +b_{1} x_{21} y_{1} .
\end{aligned}
$$

Since $e_{2 N-j}=\sum_{i=1}^{N-1} e_{2 i-j}$ for $j=0,1$, then formally only $N-1$ errors can be considered in (13).

In order to design the controls we will consider in this work the following Lyapunov function

$$
V(x)=\sum_{i=1}^{N} \frac{\alpha_{i}}{4} y_{i}^{2}+\frac{1}{2} \sum_{i=1}^{2 N} e_{i}^{2},
$$

where $\alpha_{i} \geq 0$ are weighting parameters. Notice that $V(x)=0$ for all $x \in \mathcal{A} \cap \prod_{i=1}^{N} \mathcal{W}_{2 i}$ and positive otherwise. Such a choice of Lyapunov function is very natural for our goal since it has two items: the former one characterizes stability of each oscillator, while the latter item evaluates synchronicity of the network.

\section{B. Preliminary results}

In [31] for $N=2$ and

$$
u=k \psi, k>0,
$$

e.g. $\varphi(\psi)=k \psi$ in (12), the following result has been proven using $V(x)$ :

Theorem 8. [31] The family of Brockett oscillators (11) with $N=2$ is synchronized by (15), i.e. in (11),(15) all solutions stay bounded for all $t \geq 0$ and the set $\mathcal{A}$ is globally asymptotically attractive.

The result of this theorem is a particular case of Proposition 11 given below for $N>2$. It has been observed in numerical experiments that for $N>2$ and (15) the synchronization persists, but the proof cannot be extended to the case $N>2$ since (14) is not a Lyapunov function in such a case.

Remark 9. To overcome this problem, based on the idea presented in [53], the following modification to the control law (15) can be proposed:

$$
u_{i}=k \psi_{i}+b_{i} x_{2 i} y_{i} .
$$

Since the modified control law (16) compensates the nonlinear part of (11), as a result the closed loop system becomes linear. In this case, it is trivial to show that the closed loop system (11) and (16) is globally asymptotically synchronized.

Theorem 8 guarantees global asymptotic stability of the synchronized behavior, but not the robustness. Note that the controls (15) and (16) are not bounded, then it is impossible to apply the result of Proposition 6 to prove robust stability of $\mathcal{W}$. Moreover, in many application areas, the control is bounded due to actuator limitations. With such a motivation, take a bounded version of (12), then from propositions 2 and 6 convergence of all trajectories in a vicinity of $\mathcal{W}$ immediately follows. If (12) is properly bounded then any accuracy of approaching $\mathcal{W}$ can be guaranteed, and the next result summarizes the conditions of synchronization:

Corollary 10. Let the set $\mathcal{A}$ contain all $\alpha$-and $\omega$-limit sets of (11), (12) and it is decomposable for given bounded $\varphi_{i}, i=$ $\overline{1, N}$, then the interconnection (11), (12) is synchronized, i.e. in (11), (12) all solutions stay bounded for all $t \geq 0$ and the set $\mathcal{A}$ is globally asymptotically attractive.

Proof: In the conditions of the corollary Assumption 2 is satisfied for (11), (12). The proof follows from the result of Theorem 3 since Assumption 1 is satisfied due to Proposition 6.

If we assume that (12) contains an additional perturbation $d \in \mathbb{R}^{N}$ :

$$
u_{i}(t)=\varphi_{i}\left[\psi(x(t))+d_{i}(t)\right], i=\overline{1, N},
$$

which models the connection errors and coupling imperfections, then ISS property with respect to the set $\mathcal{A}$ can be proven in the conditions of Corollary 10 (the result of Theorem 3).

\section{Global synchronization control}

Consider a variant of synchronization control in the following form:

$$
\begin{gathered}
u=k\left[\begin{array}{ccccc}
-2 & 1 & 0 & \cdots & 1 \\
1 & -2 & 1 & \cdots & 0 \\
0 & 1 & -2 & \cdots & 0 \\
\vdots & & \vdots & \ddots & \\
1 & \cdots & 0 & 1 & -2
\end{array}\right]\left[\begin{array}{c}
x_{21} \\
x_{22} \\
\vdots \\
x_{2(N-1)} \\
x_{2 N}
\end{array}\right] \\
=k S^{T}\left[\begin{array}{c}
e_{2} \\
e_{4} \\
\vdots \\
e_{2 N-2} \\
e_{2 N}
\end{array}\right],
\end{gathered}
$$

where $k>0$ is the coupling strength. Obviously, the control (17) can be rewritten as (12):

$$
u=-k S^{T} \psi
$$

With such a control each $i^{\text {th }}$ oscillator is connected with its neighbors $(i-1)^{\text {th }}$ and $(i+1)^{\text {th }}$ oscillators, and the closed loop network (11), (17) is organized again in the form of $N$-cycle graph [32]. Note that for $N=2$ the control (17) takes the form of (15).

Let us calculate the derivative of the Lyapunov function $V(x)$ for (11), (17) (in the calculations below we will use 
convention for indexes that $N+1=1$ ):

$$
\begin{aligned}
\dot{V}= & \sum_{i=1}^{N} \frac{\alpha_{i}}{2} y_{i} \dot{y}_{i}+\sum_{i=1}^{N}\left(e_{2 i-1} \dot{e}_{2 i-1}+e_{2 i} \dot{e}_{2 i}\right) \\
= & \sum_{i=1}^{N}\left[\alpha_{i}\left(-b_{i} x_{2 i}^{2} y_{i}^{2}+a_{i} x_{2 i} y_{i} u_{i}\right)\right. \\
& \left.+e_{2 i}\left(a_{i} u_{i}-a_{i+1} u_{i+1}-b_{i} x_{2 i} y_{i}+b_{i+1} x_{2(i+1)} y_{i+1}\right)\right] \\
= & \sum_{i=1}^{N}\left[a_{i}\left(\alpha_{i} x_{2 i} y_{i}+e_{2 i}-e_{2 i-2}\right) u_{i}\right. \\
& \left.+b_{i}\left(e_{2 i-2}-e_{2 i}\right) x_{2 i} y_{i}-\alpha_{i} b_{i} x_{2 i}^{2} y_{i}^{2}\right] \\
= & \sum_{i=1}^{N}\left[a_{i}\left(\alpha_{i} x_{2 i} y_{i}+e_{2 i}-e_{2 i-2}\right) k\left(e_{2 i-2}-e_{2 i}\right)\right. \\
& \left.+b_{i}\left(e_{2 i-2}-e_{2 i}\right) x_{2 i} y_{i}-\alpha_{i} b_{i} x_{2 i}^{2} y_{i}^{2}\right] \\
= & \sum_{i=1}^{N}\left[\left\{a_{i} \alpha_{i} k+b_{i}\right\}\left(e_{2 i-2}-e_{2 i}\right) x_{2 i} y_{i}\right. \\
& \left.-k a_{i}\left(e_{2 i-2}-e_{2 i}\right)^{2}-\alpha_{i} b_{i} x_{2 i}^{2} y_{i}^{2}\right] .
\end{aligned}
$$

Select $\alpha_{i}=\frac{b_{i}}{k a_{i}}$, then

$$
\begin{aligned}
\dot{V}= & \sum_{i=1}^{N} b_{i}\left[2\left(e_{2 i-2}-e_{2 i}\right) x_{2 i} y_{i}\right. \\
& \left.-\alpha_{i}^{-1}\left(e_{2 i-2}-e_{2 i}\right)^{2}-\alpha_{i} x_{2 i}^{2} y_{i}^{2}\right] \\
= & -\sum_{i=1}^{N} b_{i}\left[\alpha_{i}^{-0.5}\left(e_{2 i-2}-e_{2 i}\right)-\alpha_{i}^{0.5} x_{2 i} y_{i}\right]^{2} \\
\leq & 0
\end{aligned}
$$

Since $V$ is positive definite with respect to the set $\mathcal{A} \cap$ $\prod_{i=1}^{N} \mathcal{W}_{2 i}$, which is compact, then all trajectories in the system are globally bounded. By LaSalle's invariance principle all trajectories of the system converge to the largest invariant set in

$$
\begin{aligned}
\Omega & =\{x \in M: \dot{V}(x)=0\} \\
& =\left\{x \in M: e_{2 i-2}-e_{2 i}=\alpha_{i} x_{2 i} y_{i}, i=\overline{1, N}\right\} .
\end{aligned}
$$

Note that $u_{i}=k\left(e_{2 i-2}-e_{2 i}\right)=k \alpha_{i} x_{2 i} y_{i}=\frac{b_{i}}{a_{i}} x_{2 i} y_{i}$ in the set $\Omega$, then on that set the control performs compensation of nonlinearity as (16) and asymptotically the dynamics of synchronization errors take the form for $i=\overline{1, N}$ :

$$
\begin{aligned}
\dot{e}_{2 i-1}= & e_{2 i}, \\
\dot{e}_{2 i}= & -e_{2 i-1}+a_{i} u_{i}-a_{i+1} u_{i+1}-b_{i} x_{2 i} y_{i} \\
& +b_{i+1} x_{2(i+1)} y_{i+1} \\
= & -e_{2 i-1}
\end{aligned}
$$

and

$$
\begin{aligned}
\dot{y}_{i} & =-2 b_{i} x_{2 i}^{2} y_{i}+2 a_{i} x_{2 i} u_{i}=0, \\
\dot{x}_{1 i} & =x_{2 i}, \\
\dot{x}_{2 i} & =-x_{1 i},
\end{aligned}
$$

i.e. the norms $\left|x_{i}\right|$ and $\left|\left(e_{2 i-1}, e_{2 i}\right)\right|$ for all $i=\overline{1, N}$ become constant on $\Omega$. Therefore, the following result has been proven:
Proposition 11. For any $k>0$ in the system (11), (17) all trajectories are bounded for all $t \geq 0$ and asymptotically converge to the largest invariant set in

$$
\begin{aligned}
\Omega_{\infty}= & \left\{x \in M:\left|x_{i}\right|=\text { const }, e_{2 i-1}^{2}+e_{2 i}^{2}=\text { const },\right. \\
& x_{2(i-1)}+x_{2(i+1)}=\left(2+\alpha_{i}\left(\left|x_{i}\right|^{2}-1\right)\right) x_{2 i}, \\
& i=\overline{1, N}\} .
\end{aligned}
$$

As we can conclude, the set $\Omega_{\infty}$ includes the dynamics of interest with synchronization at the unit circle (when $\left|x_{i}\right|=1$ for all $i=\overline{1, N}$ ) or on a circle (when $\left|x_{i}\right| \neq 0$ for all $i=\overline{1, N}$ ). Indeed, the relations

$$
\beta_{i} x_{2 i}=x_{2(i-1)}+x_{2(i+1)}
$$

with constant $\beta_{i}=2+\alpha_{i}\left(\left|x_{i}\right|^{2}-1\right)$, which satisfy in the set $\Omega_{\infty}$ for all $i=\overline{1, N}$, can be interpreted as a kind of synchronization, with another synchronization measure (the previously introduced $\psi(x(t))$ may be non zero in general case). Note that different, phase or anti-phase, patterns can be obtained in (11), (17) depending on values of parameters. The case when $\left|x_{i}\right|=0$ for all $i=\overline{1, N}$ corresponds also to synchronization, but it is not interesting from application point of view since there is no oscillating solution in this case.

Theorem 12. For any $k>0$, if there is an index $1 \leq i \leq N$ such that $2 a_{i} k<b_{i}$, then in the system (11), (17) all trajectories are bounded and almost all of them converge to the largest invariant set in

$$
\begin{aligned}
\Omega_{\infty}^{\prime}= & \left\{x \in M:\left|x_{i}\right|=\text { const } \neq 0, e_{2 i-1}^{2}+e_{2 i}^{2}=\text { const },\right. \\
& x_{2(i-1)}+x_{2(i+1)}=\left(2+\alpha_{i}\left(\left|x_{i}\right|^{2}-1\right)\right) x_{2 i}, \\
& i=\overline{1, N}\} .
\end{aligned}
$$

Proof: Since all conditions of Proposition 11 are satisfied, then all trajectories converge to the set $\Omega_{\infty}$. By substitution of the control (17) in the equations of (11) we obtain:

$$
\begin{aligned}
\dot{x}_{1 i}= & x_{2 i}, \\
\dot{x}_{2 i}= & a_{i} u_{i}-x_{1 i}-b_{i} x_{2 i}\left(\left|x_{i}\right|^{2}-1\right) \\
= & a_{i} k\left(x_{2(i-1)}-2 x_{2 i}+x_{2(i+1)}\right)-x_{1 i} \\
& -b_{i} x_{2 i}\left(\left|x_{i}\right|^{2}-1\right) \\
= & -x_{1 i}-\left(2 a_{i} k-b_{i}\right) x_{2 i}+a_{i} k\left(x_{2(i-1)}\right. \\
& \left.+x_{2(i+1)}\right)-b_{i} x_{2 i}\left|x_{i}\right|^{2} .
\end{aligned}
$$

Linearizing this system around the origin $\left(\left|x_{i}\right|=0\right.$ for all $i=\overline{1, N}$ ) we conclude that this equilibrium is unstable if there exists at least one index $1 \leq i \leq N$ with $2 a_{i} k<b_{i}$. Thus, for almost all initial conditions trajectories converge to a subset of $\Omega_{\infty}$ where $\left|x_{i}\right| \neq 0$, i.e. to the set $\Omega_{\infty}^{\prime}$ (see Proposition 11 in [54]).

The result of the theorem establishes conditions for the existence of various oscillatory synchronization patterns in (11), (17). Next, let us develop additional conditions to be checked to guarantee the desired synchronization pattern with $\psi(x(t))=0$.

To this end, in the set $\Omega_{\infty}$ we have for all $i=\overline{1, N}$ :

$$
\begin{gathered}
x_{1 i}^{2}+x_{2 i}^{2}=r_{i}^{2}, \\
\rho_{i}^{2}=e_{2 i-1}^{2}+e_{2 i}^{2}=r_{i}^{2}+r_{i+1}^{2}-2\left(x_{1 i} x_{1(i+1)}+x_{2 i} x_{2(i+1)}\right)
\end{gathered}
$$


for some $r_{i} \in \mathbb{R}_{+}$and $\rho_{i} \in \mathbb{R}_{+}$, and

$$
x_{2(i-1)}+x_{2(i+1)}=\beta_{i} x_{2 i}, x_{1(i-1)}+x_{1(i+1)}=\beta_{i} x_{1 i}+c_{i}
$$

for $\beta_{i}=2+\alpha_{i}\left(r_{i}^{2}-1\right), \alpha_{i}=\frac{b_{i}}{k a_{i}}$ and some $c_{i} \in \mathbb{R}$. Note that if $b_{i}>2 a_{i} k$ then $\beta_{i}$ can take non-positive values. If $\beta_{i}=0$, then from the above equations $x_{2(i-1)}=-x_{2(i+1)}$ and $x_{1(i-1)}=c_{i}-x_{1(i+1)}$; using these relations and taking sum of $\rho_{i}^{2}$ and $\rho_{i-1}^{2}$ we obtain

$$
\rho_{i}^{2}+\rho_{i-1}^{2}=2 r_{i}^{2}+r_{i+1}^{2}+r_{i-1}^{2}-2 x_{1 i} c_{i},
$$

consequently, for $c_{i} \neq 0$ the variable $x_{1 i}$ has to be constant, which is impossible in $\Omega_{\infty}^{\prime}$, then $c_{i}=0$ leading to an equality

$$
\rho_{i}^{2}+\rho_{i-1}^{2}=2 r_{i}^{2}+r_{i+1}^{2}+r_{i-1}^{2}, r_{i}=\sqrt{1-2 \alpha_{i}^{-1}} .
$$

Assume that $\beta_{i} \neq 0$, then finding from equations (19) the expressions for $x_{1 i}$ and $x_{2 i}$ and substituting them into the equation for $\rho_{i}$ we obtain:

$$
\begin{aligned}
\rho_{i}^{2}= & r_{i}^{2}+\left(1-2 \beta_{i}^{-1}\right) r_{i+1}^{2}-2 \beta_{i}^{-1}\left(x_{1(i-1)} x_{1(i+1)}\right. \\
& \left.+x_{2(i-1)} x_{2(i+1)}-c_{i} x_{1(i+1)}\right) .
\end{aligned}
$$

Taking square of both sides in (19) and adding them we get:

$$
\begin{aligned}
\beta_{i}^{2} r_{i}^{2}+c_{i}\left(2 \beta_{i} x_{1 i}+c_{i}\right)= & r_{i-1}^{2}+r_{i+1}^{2}+2\left(x_{1(i-1)} x_{1(i+1)}\right. \\
& \left.+x_{2(i-1)} x_{2(i+1)}\right),
\end{aligned}
$$

from which the expression for $x_{1(i-1)} x_{1(i+1)}+x_{2(i-1)} x_{2(i+1)}$ can be derived and substituted in the expression for $\rho_{i}^{2}$ :

$$
\begin{aligned}
2 c_{i}\left(\beta_{i}^{-1} x_{1(i+1)}-x_{1 i}\right)= & \rho_{i}^{2}-r_{i}^{2}-\left(1-2 \beta_{i}^{-1}\right) r_{i+1}^{2} \\
& +\beta_{i}^{-1}\left(\beta_{i}^{2} r_{i}^{2}-r_{i-1}^{2}-r_{i+1}^{2}+c_{i}^{2}\right),
\end{aligned}
$$

where the right-hand side is a constant. Differentiating this equation we conclude that either $c_{i}=0$ or

$$
x_{2(i+1)}=\beta_{i} x_{2 i}
$$

that from (19) implies $x_{2(i-1)}=0$ for all $i=\overline{1, N}$. Thus, if we are interested in the solution into $\Omega_{\infty}^{\prime}$, then we have to select the option $c_{i}=0$, which leads to the set of equations

$$
\rho_{i}^{2}=\left(1-\beta_{i}\right) r_{i}^{2}+\left(1-\beta_{i}^{-1}\right) r_{i+1}^{2}+\beta_{i}^{-1} r_{i-1}^{2}
$$

or, equivalently,

$$
\begin{aligned}
\rho_{i}^{2}= & \frac{1+\alpha_{i}\left(r_{i}^{2}-1\right)}{2+\alpha_{i}\left(r_{i}^{2}-1\right)} r_{i+1}^{2}-\left(1+\alpha_{i}\left(r_{i}^{2}-1\right)\right) r_{i}^{2} \\
& +\frac{1}{2+\alpha_{i}\left(r_{i}^{2}-1\right)} r_{i-1}^{2}
\end{aligned}
$$

for all $i=\overline{1, N}$. For the subsystems with $b_{i}>2 a_{i} k$ (the solution $2+\alpha_{i}\left(r_{i}^{2}-1\right)=0$ is admissible), the corresponding equation in (21) has to be replaced with (20).

Note that the system of equations (21) for $r_{i}=1, i=\overline{1, N}$ admits the only solution $\rho_{i}=0, i=\overline{1, N}$. If we assume that $\rho_{i}=0, i=\overline{1, N}$, then by definition $r_{i}^{2}=r_{i+1}^{2}$ and (21) can be reduced to

$$
0=\alpha_{i}\left(r_{i}^{2}-1\right) r_{i}^{2}, i=\overline{1, N},
$$

which in $\Omega_{\infty}^{\prime}$ has the only admissible solution $r_{i}=1, i=$ $\overline{1, N}$, as we need. Unfortunately, the equation (21) (as well as (20)) admits also other solutions with $r_{i} \in(0,1)$ and $\rho_{i} \neq 0$.

In order to exclude other solutions with $\rho_{i} \neq 0$ let us consider a Lyapunov function

$$
W=\frac{1}{2} \sum_{i=1}^{N}\left|x_{i}\right|^{2}=\frac{1}{2} \sum_{i=1}^{N} x_{1 i}^{2}+x_{2 i}^{2},
$$

whose time derivative has the form:

$$
\begin{aligned}
\dot{W}= & -\sum_{i=1}^{N} b_{i} x_{2 i}^{2}\left(\left|x_{i}\right|^{2}-k \frac{a_{i+1}+a_{i-1}-2 a_{i}}{2 b_{i}}-1\right) \\
& -\sum_{i=1}^{N} k \frac{a_{i}+a_{i+1}}{2} e_{2 i}^{2} .
\end{aligned}
$$

According to Theorem 12, asymptotically $\dot{W}=0$ in the set $\Omega_{\infty}^{\prime}$, then

$$
\begin{gathered}
\sum_{i=1}^{N} b_{i} x_{2 i}^{2}\left(\left|x_{i}\right|^{2}-\frac{a_{i+1}+a_{i-1}-2 a_{i}}{2 b_{i} k^{-1}}-1\right) \\
+\frac{a_{i}+a_{i+1}}{2 k^{-1}} e_{2 i}^{2}=0 .
\end{gathered}
$$

Note that in the set $\Omega_{\infty}^{\prime}$ we have $x_{2 i}=r_{i} \sin \left(\phi_{i}-t\right)$ for all $i=\overline{1, N}$, where $r_{i}=\left|x_{i}\right|$ and $\phi_{i} \in[0,2 \pi)$ are some constants depending on the system parameters and initial conditions, then the equation above can be rewritten as follows:

$$
\begin{aligned}
0= & \sum_{i=1}^{N} k \frac{a_{i}+a_{i+1}}{2} r_{i} r_{i+1}\left(\cos \left(\phi_{i}-\phi_{i+1}\right)-1\right) \\
& +r_{i}\left[k\left(a_{i}+a_{i+1}\right) r_{i+1} \sin ^{2}\left(\frac{\phi_{i}+\phi_{i+1}}{2}-t\right)\right. \\
& \left.-r_{i}\left(b_{i}\left(r_{i}^{2}-1\right)+2 k a_{i}\right) \sin ^{2}\left(\phi_{i}-t\right)\right] .
\end{aligned}
$$

This equation has a trivial solution $\phi_{i}=\phi_{i+1}$ and $r_{i}=1$ for all $i=\overline{1, N}$ (the case of synchronization). Differentiating this equality, we obtain:

$$
\begin{aligned}
0= & \sum_{i=1}^{N} k\left(a_{i}+a_{i+1}\right) r_{i} r_{i+1} \sin \left(\phi_{i}+\phi_{i+1}-2 t\right) \\
& -r_{i}^{2}\left(b_{i}\left(r_{i}^{2}-1\right)+2 k a_{i}\right) \sin \left(2 \phi_{i}-2 t\right),
\end{aligned}
$$

and differentiating once more:

$$
\begin{aligned}
0= & \sum_{i=1}^{N} k\left(a_{i}+a_{i+1}\right) r_{i} r_{i+1}\left(1-2 \sin ^{2}\left(\frac{\phi_{i}+\phi_{i+1}}{2}-t\right)\right) \\
& -r_{i}^{2}\left(b_{i}\left(r_{i}^{2}-1\right)+2 k a_{i}\right)\left(1-2 \sin ^{2}\left(\phi_{i}-t\right)\right) .
\end{aligned}
$$

Finally combining (22) and (23), we derive a time-invariant equation

$$
\begin{aligned}
0= & \sum_{i=1}^{N} k\left(a_{i}+a_{i+1}\right) r_{i} r_{i+1} \cos \left(\phi_{i}-\phi_{i+1}\right) \\
& -r_{i}^{2}\left(b_{i}\left(r_{i}^{2}-1\right)+2 k a_{i}\right),
\end{aligned}
$$

which describes all possible relations between $\phi_{i}$ and $r_{i}$ for $i=\overline{1, N}$ such that the corresponding trajectories are in $\Omega_{\infty}^{\prime}$. Note that by definition:

$$
\rho_{i}^{2}=r_{i}^{2}+r_{i+1}^{2}-2 r_{i} r_{i+1} \cos \left(\phi_{i}-\phi_{i+1}\right),
$$


then we obtain

$$
\begin{aligned}
0= & \sum_{i=1}^{N}\left(\rho_{i}^{2}-r_{i}^{2}-r_{i+1}^{2}\right) k\left(a_{i}+a_{i+1}\right) \\
& +2 r_{i}^{2}\left(b_{i}\left(r_{i}^{2}-1\right)+2 k a_{i}\right),
\end{aligned}
$$

which together with $N$ equations in (21) form the system of $N+1$ nonlinear algebraic equations for $2 N$ unknowns $\left(r_{i}\right.$ and $\rho_{i}$ ) describing the kind of synchronization in (11), (17) that is admissible in $\Omega_{\infty}^{\prime}((24)$ is not a linear combination of (21)).

Corollary 13. Let all conditions of Theorem 12 be satisfied, and all solutions of (21), (24) with $r_{i} \neq 1$ admit the restriction:

$$
r_{i}^{2}<\frac{1}{3}\left(1-2 \frac{k a_{i}}{b_{i}}\right)
$$

for some $1 \leq i \leq N$. Then for almost all initial conditions the system (11), (17) is synchronized.

As we can note, $b_{i}>2 a_{i} k$ is a necessary condition for Corollary 13 to satisfy.

Proof: Let us consider the dynamics of the variables $r_{i}$ :

$$
\dot{r}_{i}=x_{2 i} \frac{a_{i} k\left(x_{2(i-1)}+x_{2(i+1)}\right)-x_{2 i}\left(b_{i}\left[\left|x_{i}\right|^{2}-1\right]+2 a_{i} k\right)}{r_{i}},
$$

then considering only trajectories in $\Omega_{\infty}^{\prime}$ and substituting $x_{2 i}=r_{i} \sin \left(\phi_{i}-t\right)$ we obtain (the same equation can be derived considering (11), (17) in polar coordinates $r_{i}$ and $\theta_{i}$ (the amplitude $r_{i}=\sqrt{x_{1 i}^{2}+x_{2 i}^{2}}$ and phase $\theta_{i}=\arctan \left(\frac{x_{2 i}}{x_{1 i}}\right)$ of an oscillator) and selecting $\theta_{i}=\phi_{i}-t$ ):

$$
\begin{gathered}
\dot{r}_{i}=a_{i} k \sin \left(\phi_{i}-t\right)\left(r_{i-1} \sin \left(\phi_{i-1}-t\right)+r_{i+1} \sin \left(\phi_{i+1}-t\right)\right) \\
-r_{i} \sin ^{2}\left(\phi_{i}-t\right)\left(b_{i}\left[r_{i}^{2}-1\right]+2 a_{i} k\right) .
\end{gathered}
$$

For any constant values $r_{i}$ and $\phi_{i}$, which constitute a solution of the system of equations (21) and (24), introduce linearization of the dynamics of $r_{i}$ taking $\phi_{i}$ as constants:

$$
\begin{gathered}
\dot{\delta r_{i}}=-\delta r_{i} \sin ^{2}\left(\phi_{i}-t\right)\left(b_{i}\left[3 r_{i}^{2}-1\right]+2 a_{i} k\right) \\
+a_{i} k \sin \left(\phi_{i}-t\right)\left(\delta r_{i-1} \sin \left(\phi_{i-1}-t\right)+\delta r_{i+1} \sin \left(\phi_{i+1}-t\right)\right),
\end{gathered}
$$

where $\delta r_{i}$ represents the deviation with respect to $r_{i}$ for $i^{\text {th }}$ oscillator in the linearized dynamics. Let us investigate a Lyapunov function showing instability of this time-varying system in the given equilibrium:

$$
U\left(\delta r_{1}, \ldots, \delta r_{N}\right)=\frac{1}{2} \sum_{i=1}^{N} \delta r_{i}^{2},
$$

then

$$
\begin{aligned}
\dot{U}= & \sum_{i=1}^{N} a_{i} k \delta r_{i} \sin \left(\phi_{i}-t\right)\left\{\delta r_{i-1} \sin \left(\phi_{i-1}-t\right)\right. \\
& \left.+\delta r_{i+1} \sin \left(\phi_{i+1}-t\right)\right\} \\
& -\delta r_{i}^{2} \sin ^{2}\left(\phi_{i}-t\right)\left(b_{i}\left[3 r_{i}^{2}-1\right]+2 a_{i} k\right) \\
= & \sum_{i=1}^{N} k\left[a_{i}+a_{i+1}\right] \delta r_{i} \sin \left(\phi_{i}-t\right) \delta r_{i+1} \sin \left(\phi_{i+1}-t\right) \\
& -\delta r_{i}^{2} \sin ^{2}\left(\phi_{i}-t\right)\left(b_{i}\left[3 r_{i}^{2}-1\right]+2 a_{i} k\right) .
\end{aligned}
$$

It is easy to check that if the condition (25) is satisfied for some $1 \leq i \leq N$ and $\delta r_{j}=0$ for all $1 \leq j \neq i \leq N$, then $\dot{U}(t)>0$ for almost all instants of time $t \geq t_{0} \geq 0$, which implies instability of the linearized dynamics. Applying the same Lyapunov function $U$ to the original nonlinear system it is possible to prove its local instability at that equilibrium point. Finally, if $b_{i}\left[3 r_{i}^{2}-1\right]+2 a_{i} k<0$ (under the condition (25)), then $b_{i}\left[r_{i}^{2}-1\right]+2 a_{i} k<0$ and the result follows Proposition 11 in [54].

\section{EXAMPLES AND SIMULATIONS}

To illustrate the theoretical results, we will consider $N=4$ non-identical Brockett oscillators in (11) with parameters

$$
a_{i}=\frac{i}{4}, b_{i}=i, i=1,2,3,4, k=1,
$$

and global synchronizing control

$$
\begin{aligned}
u & =k\left[\begin{array}{cccc}
-2 & 1 & 0 & 1 \\
1 & -2 & 1 & 0 \\
0 & 1 & -2 & 1 \\
1 & 0 & 1 & -2
\end{array}\right]\left[\begin{array}{l}
x_{21} \\
x_{22} \\
x_{23} \\
x_{24}
\end{array}\right], \\
& =k\left[\begin{array}{cccc}
-1 & 0 & 0 & 1 \\
1 & -1 & 0 & 0 \\
0 & 1 & -1 & 0 \\
0 & 0 & 1 & -1
\end{array}\right]\left[\begin{array}{l}
e_{2} \\
e_{4} \\
e_{6} \\
e_{8}
\end{array}\right] .
\end{aligned}
$$

With such a control each $i^{\text {th }}$ oscillator is connected with its neighbors $(i-1)^{\text {th }}$ and $(i+1)^{\text {th }}$ oscillators, and the closed loop network (11), (26) is organized in the form of $N$-cycle graph. The chosen parameters respect the necessary condition $b_{i}>2 a_{i} k$ of Corollary 13 for all $i=\overline{1, N}$. In order to check (25) the system of equations (21), (24) was solved using a Newton iterative method for 1000 random initial conditions. If the norm of the error in the equations (21), (24) on the last step was less than 0.1 , then it was assumed that a solution to (21), (24) has been found and (25) was tested for the found values of $r_{i}$, and (25) was always verified. Then, according to Corollary 13, the system (11), (17) is synchronized and it converges to the unit circle. Select the initial conditions as $(-3,0),(0,-3),(3,0)$ and $(0,3)$. With the selected initial conditions, the result of the simulation with controller (26) can be found in Fig. 1. From this figure, it can be seen that the oscillators are synchronized in the unit circle as predicted by Corollary 13. This demonstrate the effectiveness of the proposed synchronization protocol.

To check the global nature of the synchronization protocol, further simulation studies are considered. In this case, the simulation ran for 50 times with randomly distributed initial conditions within the range $(-5,5)$. The errors among the state variables can be seen in Fig. 2 and the histogram of the initial conditions distribution can be seen in Fig. 3. The plot of Fig. 2 clearly shows that the global synchronization is achieved and proposed synchronization protocol is not dependent on the initial conditions.

To the best of our knowledge, the literature on the synchronization of multi-stable systems is very limited and we do not know an existing result that can be applied for Brockett 


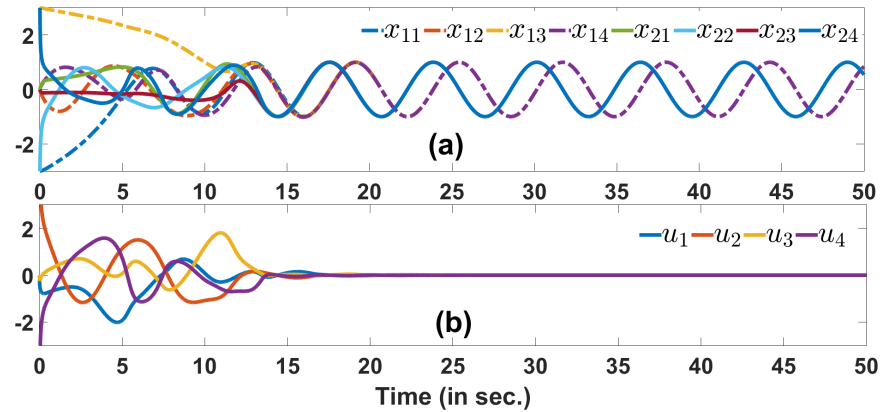

Figure 1. The results of simulation of (11), (26) for $N=4$. a) Evolution of the oscillator states; Bottom) Control signals.

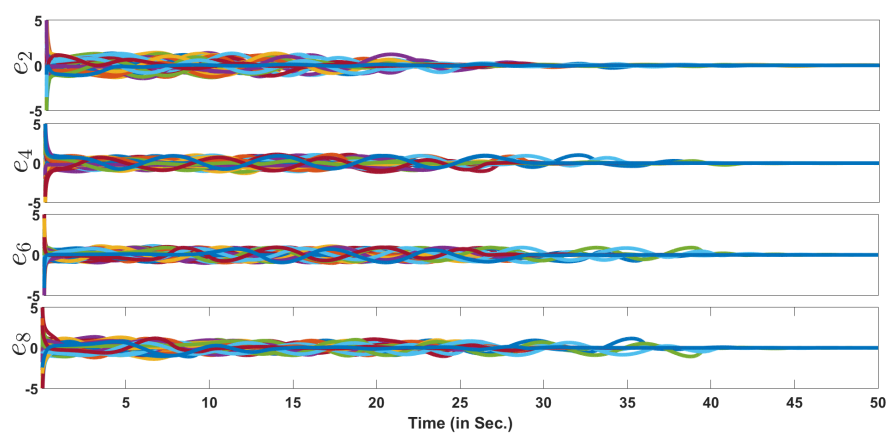

Figure 2. Evolution of errors $e_{2 i}=x_{2 i}-x_{2(i+1)}, i=\overline{1,4}$ with randomly selected initial conditions for 50 simulations run.

oscillators guaranteeing a global synchronization in this nonlinear system. Alternatively, the nonlinearities of the Brockett oscillator can be considered as a disturbance or an unknown input. Then following the idea of [53], a feedback linearizing controller can be designed. However, the closed-loop system in such a context becomes linear and it is not possible to investigate an oscillatory synchronization. Our proposed synchronization protocol can guarantee the convergence to the unit-circle through Corollary 13. This is a considerable advantage over the existing literature.

\section{CONCLUSiOnS}

This paper studied the problem of global robust synchronization of non-identical Brockett oscillators. To this end, global stability and ISS analysis were done for an individual oscillator (with respect to the set $\mathcal{W}$ composed by the equi-

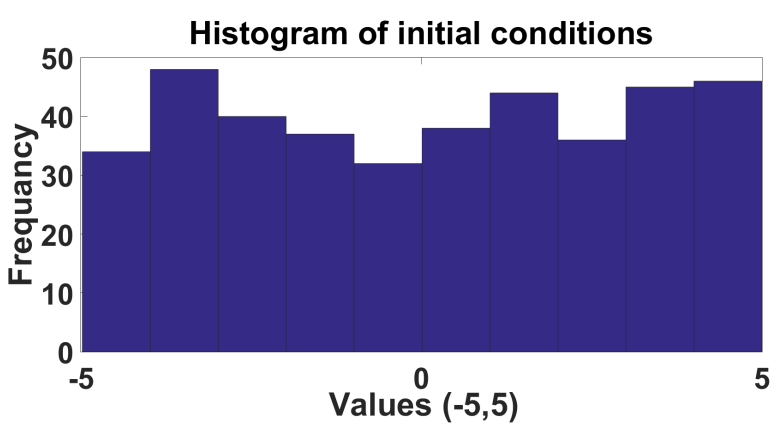

Figure 3. Histogram of initial conditions distributions. librium at the origin and the limit cycle at the unit sphere). These results make Brockett oscillator a promising benchmark model for the investigation of synchronization and consensus phenomena. Next, two synchronization control strategies were proposed. The first one imposes restriction on the synchronization control amplitude and uses generic ISS arguments. The second synchronization control design is based on a special Lyapunov function proposed in this work, and it allows the kind of synchronous motions to be evaluated. Numerical simulations demonstrated the effectiveness of our method by applying it to networks of non-identical and identical Brockett oscillators.

\section{REFERENCES}

[1] R. Brockett, "Synchronization without periodicity," in Mathematical Systems Theory, A Volume in Honor of U. Helmke, K. Huper and J. Trumpf, Eds. CreateSpace, 2013, pp. 65-74.

[2] A. Isidori, L. Marconi, and G. Casadei, "Robust output synchronization of a network of heterogeneous nonlinear agents via nonlinear regulation theory," IEEE Transactions on Automatic Control, vol. 59, no. 10, pp. 2680-2691, 2014.

[3] G. Casadei, A. Isidori, and L. Marconi, "About disconnected topologies and synchronization of homogeneous nonlinear agents over switching networks," International Journal of Robust and Nonlinear Control, 2017.

[4] A. V. Proskurnikov, "Average consensus in networks with nonlinearly delayed couplings and switching topology," Automatica, vol. 49, no. 9, pp. 2928-2932, 2013.

[5] H. Du, G. Wen, Y. Cheng, Y. He, and R. Jia, "Distributed finite-time cooperative control of multiple high-order nonholonomic mobile robots," IEEE Transactions on Neural Networks and Learning Systems, vol. PP, no. 99 , pp. 1-9, 2017.

[6] M. Defoort, T. Floquet, A. Kokosy, and W. Perruquetti, "Sliding-mode formation control for cooperative autonomous mobile robots," IEEE Transactions on Industrial Electronics, vol. 55, no. 11, pp. 3944-3953, 2008.

[7] H. Du and S. Li, "Attitude synchronization for flexible spacecraft with communication delays," IEEE Transactions on Automatic Control, vol. 61, no. 11, pp. 3625-3630, Nov 2016.

[8] I. I. Blekhman, Synchronization in science and technology. ASME Press, 1988.

[9] G. V. Osipov, J. Kurths, and C. Zhou, Synchronization in Oscillatory Networks. Springer, 2007.

[10] A. Pikovsky and J. Kurths, Synchronization: A Universal Concept in Nonlinear Sciences. Cambridge University Press, 2003.

[11] S. H. Strogatz, Sync: How Order Emerges from Chaos in the Universe, Nature, and Daily Life. Hyperion, 2004.

[12] E. M. Izhikevich, Dynamical systems in neuroscience. MIT press, 2007.

[13] R. Jeter and I. Belykh, "Synchronization in on-off stochastic networks: windows of opportunity," Circuits and Systems I: Regular Papers, IEEE Transactions on, vol. 62, no. 5, pp. 1260-1269, 2015.

[14] E. Mallada, R. A. Freeman, and A. K. Tang, "Distributed synchronization of heterogeneous oscillators on networks with arbitrary topology," IEEE Transactions on Control of Network Systems, vol. 3, no. 1, pp. 12-23, 2016.

[15] C. S. Pappu, B. C. Flores, P. S. Debroux, and J. E. Boehm, "An electronic implementation of lorenz chaotic oscillator synchronization for bistatic radar applications," IEEE Transactions on Aerospace and Electronic Systems, vol. 53, no. 4, pp. 2001-2013, 2017.

[16] R. Sepulchre, D. Paley, and N. Leonard, "Collective motion and oscillator synchronization," in Cooperative control. Springer, 2005, pp. $189-205$.

[17] F. Dorfler and F. Bullo, "Synchronization and transient stability in power networks and nonuniform Kuramoto oscillators," SIAM Journal on Control and Optimization, vol. 50, no. 3, pp. 1616-1642, 2012.

[18] B. B. Johnson, S. V. Dhople, A. O. Hamadeh, and P. T. Krein, "Synchronization of nonlinear oscillators in an LTI electrical power network," Circuits and Systems I: Regular Papers, IEEE Transactions on, vol. 61 , no. 3, pp. 834-844, 2014.

[19] S. V. Dhople, B. B. Johnson, F. Dorfler, and A. O. Hamadeh, "Synchronization of nonlinear circuits in dynamic electrical networks with general topologies," Circuits and Systems I: Regular Papers, IEEE Transactions on, vol. 61, no. 9, pp. 2677-2690, 2014. 
[20] A. Dòria-Cerezo, J. M. Olm, M. di Bernardo, and E. Nuño, "Modelling and control for bounded synchronization in multi-terminal VSC-HVDC transmission networks," IEEE Transactions on Circuits and Systems I: Regular Papers, vol. 63, no. 6, pp. 916-925, 2016.

[21] A. E. Motter, S. A. Myers, M. Anghel, and T. Nishikawa, "Spontaneous synchrony in power-grid networks," Nature Physics, vol. 9, no. 3, p. 191, 2013.

[22] E. Oviedo, N. Vazquez, and R. Femat, "Synchronization technique of grid-connected power converters based on a limit cycle oscillator," IEEE Transactions on Industrial Electronics, vol. 65, no. 1, pp. 709-717, 2018.

[23] G. Cena, I. C. Bertolotti, S. Scanzio, A. Valenzano, and C. Zunino, "Synchronize your watches: Part I: General-purpose solutions for distributed real-time control," Industrial Electronics Magazine, IEEE, vol. 7, no. 1, pp. 18-29, 2013.

[24] S. Knorn, Z. Chen, and R. H. Middleton, "Overview: Collective control of multiagent systems," IEEE Transactions on Control of Network Systems, vol. 3, no. 4, pp. 334-347, 2016.

[25] V. Gazi and K. M. Passino, Swarm Stability and Optimization. Springer, 2011.

[26] J. S. Shamma, Cooperative Control of Distributed Multi-Agent Systems. Wiley-Interscience, 2008.

[27] R. Olfati-Saber, "Flocking for multi-agent dynamic systems: algorithms and theory," Automatic Control, IEEE Transactions on, vol. 51, no. 3, pp. 401-420, 2006.

[28] F. Lewis, H. Zhang, K. Hengster-Movric, and A. Das, Cooperative Control of Multi-Agent Systems, ser. Communications and Control Engineering. Springer, 2014.

[29] W. Ren, R. W. Beard, and E. M. Atkins, "Information consensus in multivehicle cooperative control," IEEE Control systems magazine, vol. 2, no. 27, pp. 71-82, 2007.

[30] R. Olfati-Saber, A. Fax, and R. M. Murray, "Consensus and cooperation in networked multi-agent systems," Proceedings of the IEEE, vol. 95, no. 1 , pp. $215-233,2007$

[31] H. Ahmed, R. Ushirobira, and D. Efimov, "On the robust synchronization of brockett oscillators," IFAC-PapersOnLine, vol. 49, no. 14, pp. 142 147, 2016.

[32] S. Pemmaraju and S. Skiena, "Cycles, stars, and wheels," Computational Discrete Mathematics Combinatiorics and Graph Theory in Mathematica, pp. 284-249, 2003.

[33] D. Angeli and D. Efimov, "Characterizations of input-to-state stability for systems with multiple invariant sets," Automatic Control, IEEE Transactions on, vol. 60, no. 12, pp. 3242-3256, 2015.

[34] S. Dashkovskiy, D. Efimov, and E. Sontag, "Input to state stability and allied system properties," Automation and Remote Control, vol. 72, no. 8, pp. 1579-1614, 2011.

[35] D. Angeli, J. Ferrell, and E. Sontag, "Detection of multistability, bifurcations and hysteresis in a large class of biological positive-feedback systems," Proc. Natl. Acad. Sci. USA, vol. 101, pp. 1822-1827, 2004.

[36] D. Angeli and E. Sontag, "Multi-stability in monotone input/output systems," Systems \& Control Lett., vol. 51, pp. 185-202, 2004.

[37] A. Gelig, G. Leonov, and V. Yakubovich, Stability of nonlinear systems with non unique equilibrium. Moscow: Nauka, 1978, [in Russian]

[38] G.-B. Stan and R. Sepulchre, "Analysis of interconnected oscillators by dissipativity theory," IEEE Trans. Automatic Control, vol. 52, pp. 256270, 2007.

[39] M. Chaves, T. Eissing, and F. Allgower, "Bistable biological systems: A characterization through local compact input-to-state stability," IEEE Trans. Automatic Control, vol. 45, pp. 87-100, 2008.

[40] A. Rantzer, "A dual to Lyapunov's stability theorem," Syst. Control Lett., vol. 42, pp. 161-168, 2001

[41] D. Angeli, "An almost global notion of input-to-state stability," IEEE Trans. Automatic Control, vol. 49, pp. 866-874, 2004.

[42] D. Efimov, "Global Lyapunov analysis of multistable nonlinear systems," SIAM Journal on Control and Optimization, vol. 50, no. 5, pp. 31323154, 2012.

[43] Z. Nitecki and M. Shub, "Filtrations, decompositions, and explosions," American Journal of Mathematics, vol. 97, no. 4, pp. 1029-1047, 1975

[44] E. Egorov and A. Koronovskii, "Dynamical control in multistable systems," Technical Physics Letters, vol. 30, no. 3, pp. 186-189, 2004.

[45] H. Ahmed, R. Ushirobira, D. Efimov, and W. Perruquetti, "Robust synchronization for multistable systems," IEEE Transactions on Automatic Control, vol. 61, no. 6, pp. 1625-1630, 2016.

[46] P. Forni and D. Angeli, "The ISS approach to the stability and robustness properties of nonautonomous systems with decomposable invariant sets: an overview," European Journal of Control, vol. 30, pp. 50-60, 2016.

[47] J. P. LaSalle, "Some extensions of Liapunov's second method," Circuit Theory, IRE Transactions on, vol. 7, no. 4, pp. 520-527, 1960.
[48] N. G. Chetaev, The stability of motion. Pergamon Press, 1961

[49] J. Lofberg, "Yalmip : A toolbox for modeling and optimization in MATLAB," in Proceedings of the CACSD Conference, Taipei, Taiwan, 2004. [Online]. Available: http://users.isy.liu.se/johanl/yalmip

[50] Z. Li, Z. Duan, G. Chen, and L. Huang, "Consensus of multiagent systems and synchronization of complex networks: A unified viewpoint," Circuits and Systems I: Regular Papers, IEEE Transactions on, vol. 57, no. 1, pp. 213-224, 2010.

[51] J. Zhao, D. Hill, and T. Liu, "Global bounded synchronization of general dynamical networks with nonidentical nodes," Automatic Control, IEEE Transactions on, vol. 57, no. 10, pp. 2656-2662, 2012.

[52] A. Y. Pogromsky, "A partial synchronization theorem," Chaos, vol. 18, p. $037107,2008$.

[53] A. Das and F. L. Lewis, "Distributed adaptive control for synchronization of unknown nonlinear networked systems," Automatica, vol. 46, no. 12, pp. 2014-2021, 2010

[54] P. Monzón and R. Potrie, "Local and global aspects of almost global stability," in Proc. 45th IEEE Conf. on Decision and Control, San Diego, USA, 2006, pp. 5120-5125.

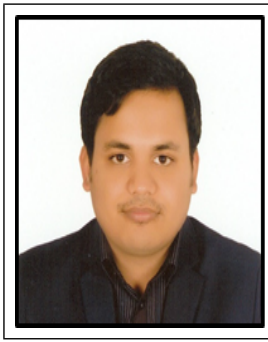

Hafiz Ahmed (S'10, M'17) received the B.Sc. degree in Electrical \& Electronic Engineering from Ahsanullah University of Science and Technology, Bangladesh, in 2011, the M.Sc. degree in Systems, Control and Information Technology from Joseph Fourier University, Grenoble, France, in 2013, and the Ph.D. degree in Automatic Control from the University of Lille 1, France, in 2016. He has obtained the Best $\mathrm{PhD}$ Theses award from the Research Cluster on Modeling, Analysis and Management of Dynamic Systems (GDR-MACS) of the National Council of Scientific Research (CNRS) in France. He was a Postdoctoral fellow at Clemson University, SC, USA followed by academic appointments in Bangladesh at the University of Asia Pacific and North South University. Since 2017, he joined the School of Mechanical, Aerospace and Automotive Engineering, Coventry University, United Kingdom. He is interested in applied control engineering with special focus in energy and environment.

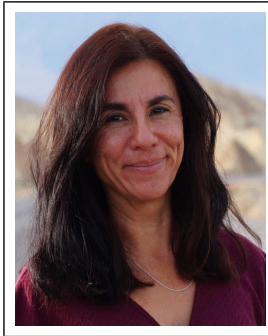

Rosane Ushirobira received her $\mathrm{PhD}$ in Mathematics from the University of Strasbourg (France) She was then a post-doctoral fellow at University of Poitiers (France) and at the Technische Universiteit Eindhoven (The Netherlands). From 1999 to 2017, she held an Associate professor position at the University of Bourgogne (France) where in 2010, she defended her Habilitation thesis. Her original research field is the Algebraic Lie theory where she has published works on Lie algebras and superalgebras, the Weyl algebra and their representations. In more recent years, she has been working at Inria (France) where she holds a Researcher position. Her main interests concern problems in Automatic Control theory, notably algebraic methods and applications in this area, and especially in estimation issues.

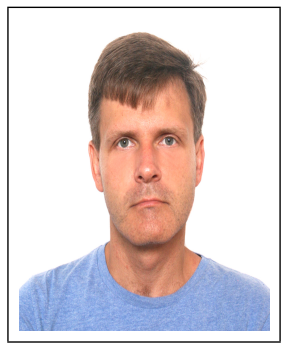

Denis Efimov (SM'11) received the Ph.D. degree in Automatic Control from the Saint-Petersburg State Electrical Engineering University (Russia) in 2001, and the Dr.Sc. degree in Automatic control in 2006 from Institute for Problems of Mechanical Engineering RAS (Saint-Petersburg, Russia). From 2000 to 2009 he was research fellow of the Institute for Problems of Mechanical Engineering RAS, Control of Complex Systems Laboratory. From 2006 to 2011 he was working in the LSS (Supelec, France), the Montefiore Institute (University of Liege, Belgium) and the Automatic control group at IMS lab (University of Bordeaux I, France). Since 2011 he joined the Non-A team at Inria Lille center. He is a member of several IFAC TCs and a Senior member of IEEE. His main research interests include oscillation analysis, observation, control and nonlinear system stability. 